\title{
Protective Effect of Oxytocin Against Bone Loss in a Female Rat Model of Osteoporosis
}

\author{
Hoda Moghazy ${ }^{1}$, Aida Abdeen Mahmoud*2, Hala Elbadre ${ }^{3}$, \\ Hekmat Osman Abdel Aziz ${ }^{4}$
}

\begin{abstract}
Background: Introduction: Oxytocin (OT) has been proposed to assist in the regulation of bone remodeling and to exert an antiosteoporotic effect. We evaluated the possible protective effect of OT against bone degeneration in ovariectomized (OVX) rats.

Methods: The study was performed on three groups of adult female rats; group I was subjected to sham operation, group II was subjected to ovariectomy, and group III was subjected to ovariectomy and intraperitoneal injection with OT for eight successive weeks. At the end of the study, bone mass density (BMD) was measured; then the rats were euthanized and their blood and bone tissues were examined.

Results: The group II rats had significantly less BMD and greater serum bone-specific alkaline phosphatase (bALP), osteocalcin (OC), and tartrate-resistant acid phosphatase (TRAP) levels than the group I rats. Furthermore, group II rats had fewer osteocytes and osteoblasts, and less OPG/RANKL mRNA expression than group I rats. The groups I and III and rats showed no significant differences in BMD, bALP, OC, TRAP, OPG/RANKL mRNA expression, or osteocyte and osteoblast numbers.
\end{abstract}

Conclusions: Oxytocin may have an antiosteoporotic effect in OVX rats.

Keywords: Osteoporosis, OPG, Oxytocin, Ovariectomy, RANKL.

\section{Introduction}

Osteoporosis is found worldwide; approximately $10 \%$ of the world's population and $30 \%$ of postmenopausal women suffer from the disease (1-2). Osteoporosis is characterized by low bone mass and disruption of bone microarchitecture (3), which lead to fractures and impaired bone regeneration (4). Despite the availability of many lines of treatment, annual hip fracture rates due to osteoporosis are expected to exceed six million by 2050 (5).

Oxytocin (OT), a polypeptide hormone synthesized in the supraoptic and paraventricular nuclei of the hypothalamus, is secreted through the posterior pituitary. Oxytocin is well recognized for its effects on parturition and lactation and has recently been shown to be involved in energy regulation and bone homeostasis (6). Oxytocin receptors are represented in many tissues including pituitary, ovary, testis, kidney, bone, muscle, pancreas, fat, thymus, heart, and vascular endothelium (7). Oxytocin has anabolic effects on bone, increasing osteoblastic over osteoclastic activity (8). In mice, OT receptors (OTRs) were found on osteoblasts and osteoclasts; in addition, the anabolic effects of estrogen on bone mass were partially mediated through OT (9). Oxytocin- and OTR-null mice suffered from severe osteoporosis with low bone turnover (10), and the administration of OT increased bone formation and improved bone microarchitecture (8). Bone resorption was found unaffected in OT deficiency because, while OT 
stimulates the genesis of osteoclasts, it inhibits their resorptive function. Furthermore, OT is produced by bone marrow osteoblasts and acts as paracrineautocrine regulator of bone formation modulated by estrogens. In turn, the power of estrogen to increase bone mass is OTR-dependent (11).

In humans, osteoporosis and reduced posteroanterior and lateral spine bone density in postmenopausal women were associated with lower serum OT levels (12-13).

Bone remodeling is mediated by osteoblasts and osteoclasts which maintain bone metabolic balance and integrity. Many cytokines, including receptor activators of nuclear factor $\mathrm{\kappa B}$ ligand (RANKL) and osteoprotegerin (OPG), are involved in regulating osteoblasts and osteoclasts; of these, osteoblasts are one of the principal sources of RANKL and OPG in bone tissues. RANKL binds to the receptor activator of nuclear factor $\kappa \mathrm{B}(\mathrm{RANK})$, present in osteoclast precursor membranes, inducing their differentiation through the ultimate activation of transcription factor NFATc-1, which modulates the expression of the genes necessary for the formation of mature osteoclast responsible for bone resorption. Osteoprotegerin acts as a decoy receptor for RANKL, thus blocking the possible interaction between RANKL and its receptor RANK (14-16).

This work aimed to study the protective effect of OT against OVX-induced bone loss through assessing bone turnover markers and expression of the two bone remodeling factors OPG and RANKL in OVX adult female rats, to assess serum OT levels, and to characterized the relationship between serum OT and bone status.

\section{Materials and methods Animal preparation}

Sixty 200-250 g 90-100-day-old adult female Sprague-Dawley rats were maintained at room temperature with natural light-dark cycle, and ad libitum access to standard chow diet and tap water. All animals were housed in groups of two in $20 \times 32 \times 20 \mathrm{~cm}$ metal box cages. Animals were maintained one week for accommodation. This study was performed in accordance with the guidelines of the University Animal Ethics and approved by the Research Ethics Committee considering care and use of laboratory animals (ethical approval number: SOH-IACUC17050308). Animals were obtained from the animal facility, Sohag University, Egypt.

\section{Experimental design and drugs}

Rats were randomly divided into 3 groups $(n=20)$ : Group I rats were sham-operated. They had the same incision as in ovariectomy, but the ovaries were not excised. Group II (OVX) and group III $(\mathrm{OVX}+\mathrm{OT})$ rats were bilaterally ovariectomized through two dorsolateral skin incisions as previously described. All rats were left for one weeks for recovery from surgery (17); then rats of group I and II were injected daily intraperitoneally (IP) with $0.1 \mathrm{ml} / \mathrm{kg} /$ day $\mathrm{NaCl} 0.9 \%$ for eight successive weeks. Group III rats were injected daily were anesthetized by intraperitoneal injection (IP) with OT $(0.1 \mathrm{mg} / \mathrm{kg} /$ day $)$ (18) for eight successive weeks. All animals were injected with ceftriaxone at a dose of $50 \mathrm{mg} / \mathrm{kg}$ immediately before and 24 and $48 \mathrm{~h}$ after surgery, with postoperative care of the surgical incision.

\section{BMD Measurement}

Rats were anesthetized by IP injection of 40 $\mathrm{mg} / \mathrm{kg}$ thiopental sodium and BMD was measured in each rat's right femur using a dualenergy x-ray absorptiometry (DXA) machine (Lunar iDXA machine). Measurements were obtained by proning the mice with the knees flexed and hips extended. Individual scans were stored, and analyzed later.

\section{Blood sample collection:}

At the end of the study, all rats were anesthetized and then euthanized. Two-three $\mathrm{ml}$ of fresh blood were collected, incubated in a 37 ${ }^{\circ} \mathrm{C}$ water bath for $10 \mathrm{~min}$, and centrifuged to isolate the serum. The serum was stored at -20 ${ }^{\circ} \mathrm{C}$ until the time of biochemical analysis.

Bone turnover biochemical marker (bALP, OC, and TRAP), estradiol, and OT assays

Commercially available ELIZA kits were used for assay of bALP (DALP-250, BioAssay Systems, CA, USA), OC (MBS728975), TRAP (MBS008723), Estradiol (MBS843353) and OT (WKEA Med. supplies, Changchun, China) assays. 


\section{RNA extraction and quantitative real time PCR}

The left femur of each animal was dissected, rapidly placed in liquid nitrogen and bone tissues were then ground into a powder using a pestle. The total RNA was extracted from bone powder using TRIzol ${ }^{\circledR}$ reagent and reverse transcribed to cDNA using a High Capacity cDNA Reverse Transcription Kit (\#K0251; Thermoscientific, Lithuania); according to manufacturer's instructions. The primers used were as shown in
Table 1. Products were amplified using a Stepone $^{\mathrm{TM}}$ real-time PCR apparatus (Applied Biosystems $^{\mathrm{TM}}$ ) under the following cycling conditions: $95{ }^{\circ} \mathrm{C}$ denaturation step for $2 \mathrm{~min}$ followed by 40 cycles of $95{ }^{\circ} \mathrm{C}$ for $15 \mathrm{sec}, 60{ }^{\circ} \mathrm{C}$ annealing and extension for $60 \mathrm{sec}$. The housekeeping gene GAPDH was used to normalize the quantities of the target genes. Data were analyzed using comparative $2^{-\Delta . \Delta . \mathrm{CT}}$ method.

Table 1. Primers used for OPG and RANKL gene expression assay.

\begin{tabular}{llllc}
\hline Gene & Accession No. & Forward primer & Reverse primer & $\begin{array}{c}\text { Product } \\
\text { length }\end{array}$ \\
\hline OPG & NM_012870.2 & GCCAACACTGATGGAGCAGAT & TCTTCATTCCCACCAACTGATG & 85 \\
RANKL & XM_008770928.2 & GCTCACCTCACCATCAATGCT & GGTACCAAGAGGACAGACTGACTTTA & 70 \\
GAPDH & XM_017604885.1 & AAATTCCATGGCACCGTCAA & AGGGATCTCGCTCCTGGAA & 85 \\
\hline
\end{tabular}

\section{Histological study}

At the end of the experiment, the left tibias from all animals were examined by histology. Proximal end of each tibia was surgically removed with a very sharp blade and the tibias were fixed, decalcified, and dehydrated. The bone tissue was then processed to prepare 5mm thick paraffin sections with a microtome (Leica RM 2035). All cut sections were stained with hematoxylin and eosin (H\&E) and the stained samples were histologically examined by light microscopy. Measurements were taken using an image analyzer (Leica version 3.7.2005-2010). Osteoblasts and osteocytes lining the endosteum were counted at $400 \mathrm{X}$ magnification from three different locations on each slide.

\section{Statistical analyses}

Data were analyzed using the SPSS version 20 software. Data are presented as means \pm SD or as $\mathrm{R}$ coefficients with associated $\mathrm{p}$ value. Variables were assessed for normality using the Shapiro-Wilk test. Data were also analyzed by ANOVA followed by the least significant difference (LSD) test. Pearson correlation coefficients were used to investigate the association between OT levels and OPG/RANKL mRNA expression in rats of groups II and III. P values less than 0.05 were considered significant.

\section{Results}

$B M D$, biochemical markers of bone turnover (bALP, OC and TRAP), OT and estradiol (E2)

Bone mass densities for all groups are shown in Table 2. The BMD of group II was significantly less than those of groups I and III $(p<0.05)$. The difference between groups I and III was not significant.

Serum E2 levels were significantly lower in group II and III compared to group I ( $p<0.05)$, indicating the effectiveness of ovariectomy procedure.

After nine weeks the group II rats had greater bALP, OC, and TRAP levels than the group I rats and significantly greater bALP and TRAP levels than the group III rats $(\mathrm{p}<0.05)$. No significant difference was observed for OC between groups I and III.

Furthermore, OT levels were significantly less in group II than in groups I and III, and also significantly less in group I than in group III $(p<0.05)$. 
OPG/RANKL gene expression levels

OPG mRNA expression was significantly greater in groups I and III than in group II ( $p<0.05)$, while
RANKL mRNA expression was significantly greater in group II than in groups I and III (Tables 3 and 4).

Table 2. Mean \pm SD of bone marrow density $\left(\mathrm{gm} / \mathrm{cm}^{2}\right)$ and serum bALP (ng/dl), osteocalcin (ng/ml), and oxytocin (ng/ml) in all groups of the experiment determined by one-way ANOVA.

\begin{tabular}{llll}
\hline $\begin{array}{l}\text { Group } \\
\text { Parameter }\end{array}$ & $\begin{array}{l}\text { Group I (sham) } \\
(\mathbf{n = 2 0})\end{array}$ & Group II $(\mathbf{O V X})(\mathbf{n = 2 0})$ & Group III $(\mathbf{O V X}+\mathbf{O T})(\mathbf{n = 2 0})$ \\
\hline BMD $\left(\mathrm{gm} / \mathrm{cm}^{2}\right)$ & $0.052 \pm 0.001$ & $0.020 \pm 0.001^{*}$ & $0.038 \pm 0.005 \#$ \\
E2 $(\mathrm{ng} / \mathrm{L})$ & $18 \pm 3.4$ & $1.9 \pm 0.4^{*}$ & $1.8 \pm 0.3^{*}$ \\
$\mathrm{bALP}(\mathrm{ng} / \mathrm{dl})$ & $53.6 \pm 1.45$ & $166.53 \pm 2.92^{*}$ & $56 \pm 6.047 \#$ \\
OC $(\mathrm{ng} / \mathrm{ml})$ & $0.65 \pm 0.037$ & $1.36 \pm 0.188^{*}$ & $0.7 \pm 0.074^{*}$ \\
TRAP $(\mathrm{U} / \mathrm{L})$ & $10 \pm 1.3$ & $16.4 \pm 1.5^{*}$ & $10.7 \pm 1.3 \#$ \\
OT $(\mathrm{ng} / \mathrm{ml})$ & $28.96 \pm 4.14$ & $15.40 \pm 2.871^{*}$ & $120.8 \pm 25.4 \#$ \\
\hline
\end{tabular}

*Significant at $\mathrm{p}<0.05, *$ Significant when compared with group I (sham), \#Significant when compared with group II (OVX).

Table 3. Mean osteocyte and osteoblast number in rat tibia sections.

\begin{tabular}{lccc}
\cline { 2 - 4 } & $\begin{array}{c}\text { Group I (sham) } \\
(\mathbf{n = 2 0})\end{array}$ & $\begin{array}{c}\text { Group II (OVX) } \\
(\mathbf{n = 2 0})\end{array}$ & $\begin{array}{c}\text { Group III (OVX + OT) } \\
(\mathbf{n = 2 0})\end{array}$ \\
\hline Osteocytes (cell/HPF) & $43 \pm 4.027$ & $6.4 \pm 0.96^{*}$ & $44 \pm 4.78^{\#}$ \\
Osteoblasts (cell/HPF) & $21.4 \pm 1.71$ & $4.5 \pm 0.71^{*}$ & $24.2 \pm 2.65^{\#}$
\end{tabular}

*Significant at $\mathrm{p}<0.05$, Significant when compared with group I (sham), \#Significant when compared with group II (OVX), HPF: high power field.

\begin{tabular}{cccc} 
& \multicolumn{3}{c}{ Table 4. OPG and RANKL gene expression in rat bone tissue. } \\
\cline { 2 - 4 } & $\begin{array}{c}\text { Group I (sham) } \\
(\mathbf{n = 2 0})\end{array}$ & $\begin{array}{c}\text { Group II (OVX) } \\
(\mathbf{n = 2 0})\end{array}$ & Group III (OVX+OT) $(\mathbf{n = 2 0})$ \\
\hline OPG & $1.04 \pm 0.096$ & $0.66 \pm 0.130^{*}$ & $0.99 \pm 0.159^{\#}$ \\
RANKL & $1.05 \pm 0.08$ & $3.25 \pm 0.390^{*}$ & $1.34 \pm 0.2^{\#}$ \\
\hline
\end{tabular}

*Significant at $\mathrm{p}<0.05$, *Significant when compared with group I (sham), \#Significant when compared with group II (OVX).

\section{Relationship between OT and OPG/RANKL gene expression levels.}

A positive relationship was found between OT and OPG gene expression in groups II and III (R $=0.56, p<0.5$ ), while a negative relationship was found between OT and RANKL gene expression in groups II and III $(R=-0.658, p<0.05)$.

\section{Histological and morphometric study.}

In the histological study, osteocytes were seen as basophilic cells inside their lacunae in the bone lamellae. Osteoblasts were relatively large and their cytoplasm was heavily basophilic.
Representative histological evidence of the bone defect seen with $H \& E$ staining supported the findings obtained by DXA and chemical assay. Group II bone showed osteoporosis as loss of normal bone architecture, and trabeculae that were thin, reduced in number and sometimes fragmented and fractured. They are separated with wide marrow spaces. A marked decrease in the number of osteocytes was associated with bone loss and appearance of cavities. The numbers of both osteocytes and osteoblasts were 


\section{Oxytocin Against Osteoporosis}

significantly less in group II than in groups I and III ( $\mathrm{p}<0.05)$ (Figs. 1 and 2, and Table 3).

In group III rats, the bone trabeculae, osteocytes and osteoblasts showed improvement. Trabecular bone appeared thicker than that of group II. The bone lamellae were mainly arranged in regular pattern. Osteocytes were apparent with large nuclei located inside lacunae the group III osteoblasts were significantly greater in number than those in group II and appeared cuboidal in shape. The matrix appeared homogenous with small cavities (Fig. 3).

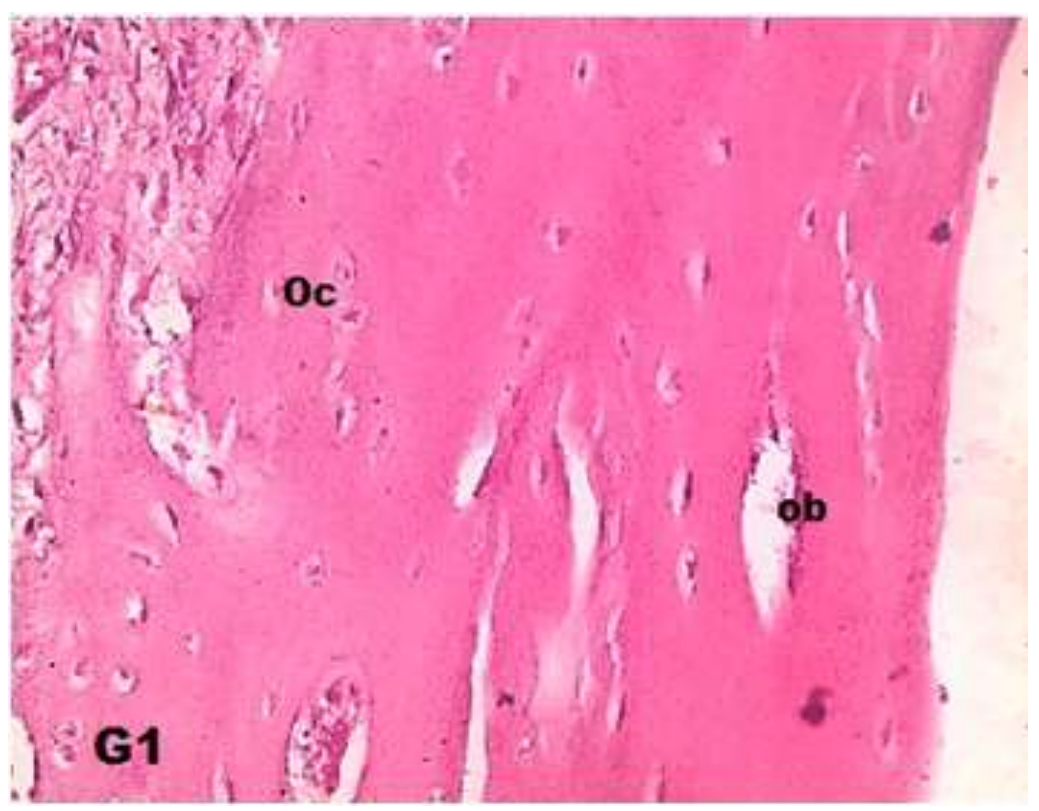

Fig.1. Photomicrograph of a section from a control rat (group I) showing the outer part of the tibial cortex. The compact bone tissue is well organized; osteocytes (Oc) inside the lacunae are shown in between the bone lamellae. Osteoblast $(\mathrm{Ob})$ are seen in the outer surface of the lamellae (H\&E X 400).

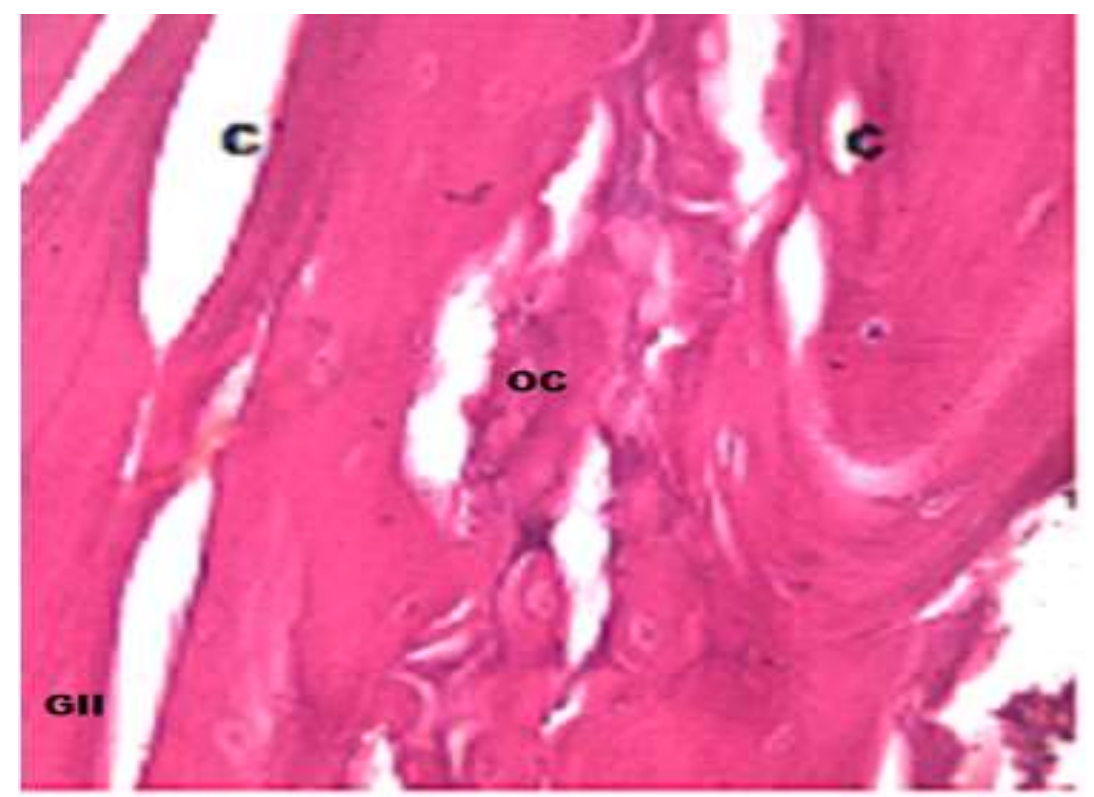

Fig.2. Photomicrograph of a section from an ovariectomized rat (group II) at the proximal end of the tibia showing cavities (C) in the bone trabeculae near the medullary cavity. The osteocyte number has also decreased (H\&E X 400). 


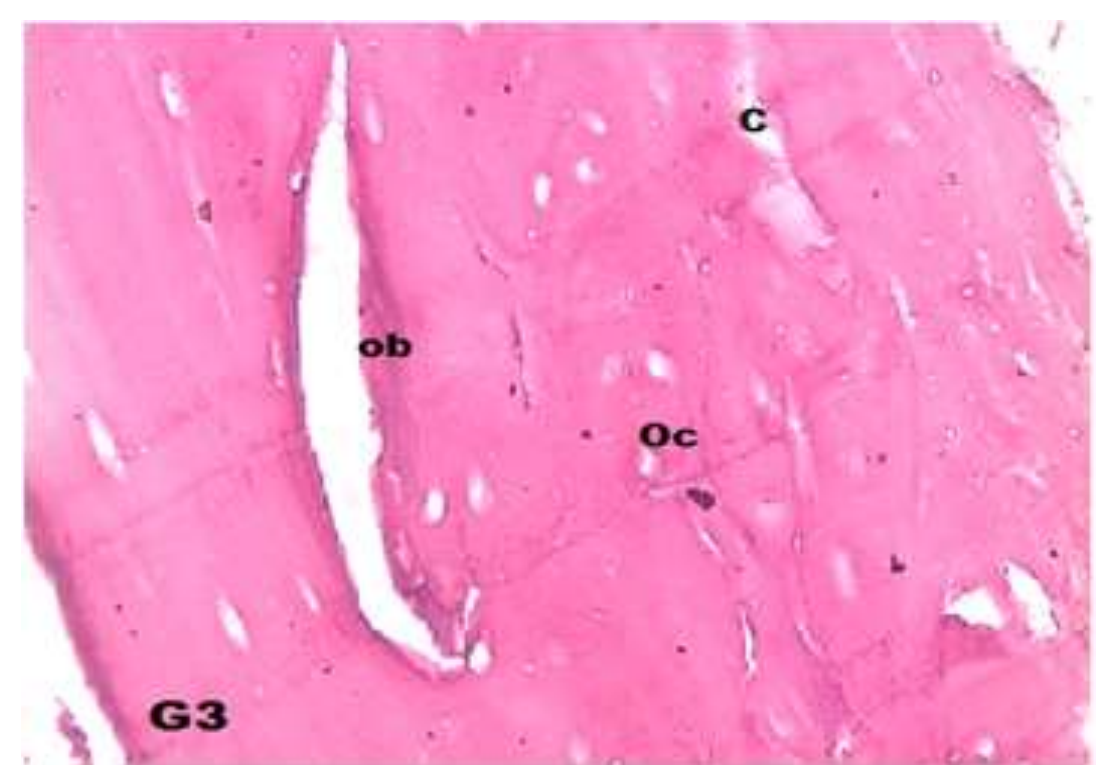

Fig. 3. Photomicrograph of a section from an ovariectomized and oxytocin-treated rat (group III) at the proximal end of the tibia showing the outer part of the bone. The cortex shows improvement and an increase in the number of osteocytes (OC) and osteoblasts (Ob). The matrix appeared homogenous with small cavities (C) (H\&E X 400).

\section{Discussion}

The increase in human life expectancy has led to an increase in age-associated problems. Osteoporosis is considered a major public health priority by the World Health Organization (WHO); however, we lack adequate preventive measures for osteoporosis complications, such as fragility fractures (19).

To mimic the natural condition occurring in postmenopausal females, bilateral ovariectomy was performed on adult female albino rats, an animal model with symptoms similar to those of early postmenopausal bone loss in humans (1). This rat model has been widely used because of its stability, reliability, high success rate, and repeatability (20).

The study revealed lower BMD and greater serum bALP, OC and TRAP in ovariectomized rats than in those in the sham group. These results agreed with previous studies that reported elevated serum ALP, OC and TRAP levels in post-menopausal females and ovariectomized rats (21-23).

Histological examination of the group II rats bone showed that the bone lost its normal architecture; the trabeculae became thin and associated with almost no osteoblastic cover with fewer number of osteocytes and osteoblasts than in the group I rats. Previous studies reported similar changes in OVX rats bones (24-26).
The bone tissue OPG mRNA expression in the OVX group in this study was significantly less than that in the sham group. This change was positively correlated with the serum OT concentration, suggesting that rat femur OPG mRNA expression may be related to changes in serum OT concentration. On the other hand, RANKL mRNA expression in OVX group femurs was significantly greater than that in the sham group. A negative correlation was seen between RANKL mRNA and OT levels in groups II and III rats. These results were in agreement with those of previous studies $(20,23)$. OPG and RANKL proteins are thought to influence postmenopausal osteoporosis; RANKL stimulates osteoclast differentiation, while OPG acts as a decoy receptor for RANKL, thus downregulating RANKL signaling through RANK and acting as an inhibitor of bone resorption (27).

Transcription of the OT and OTR genes is under the control of estrogens; therefore, as estrogen is decreased in OVX rats, as well as in postmenopausal women, OT levels are lowered as a consequence (13). It is postulated that the anabolic action of estrogen in mice occurs, at least in part, through an autocrine feed-forward OT/OTR loop exists in which estrogen releases OT from osteoblasts, which then acts upon osteoblastic OTRs to further amplify estrogen action (11). The 
bone loss in group II rats may be attributed to lack of inhibiting activity of estrogen on osteoclasts and to lack of its stimulating action on osteoblasts. This bone resorption is followed by some increase in bone formation to try to fill the high number of resorption cavities (28).

To examine the potential ability of OT to protect against bone loss induced by ovariectomy in female rats, OT treatment was started one week after OVX and continued for eight successive weeks in the group III rats. To evaluate bone condition before the start of OT treatment, BMD and serum bALP and OC were measured. These parameters were not significantly different in groups I and III. This indicates that OVX-induced bone loss did not occur, while dramatic bone loss was seen in the ovariectomized group by two weeks. At the end of the experiment, BMD and serum bALP, OC, and TRAP were not significantly different between groups 1 and III.

Histological examination at the end of the experiment showed normal bone architecture, as demonstrated by apparent maintained thickness of the bone trabecular with fewer cavitation in group III. In addition, osteocytes and osteoblasts numbers were not significantly different in groups I and III, while fewer of these cells were observed in group II than in groups I and III.

Rat bone tissue OPG and RANKL mRNA expression in bone in group III rats demonstrated that OT treatment directed expression of these genes back toward normal levels.

The mechanism that may be involved in skeletal recovery by OT was demonstrated by others. Oxytocin might exert its action by improving potential osteogenic differentiation of

\section{References}

1. Camara CE, Linyuan Z, Dong YU, Yaowu Z, Nianhong Y. Serum osteocalcin levels and bone mineral density in ovariectomized rats. International Journal of Innovation and Scientific Research. 2014;5(1): 1-8.

2. Mukaiyama K, Kamimura M, Uchiyama S, Ikegami S, Nakamura Y, Kato H. Elevation of serum alkaline phosphatase (ALP) level in postmenopausal women is caused by high bone turnover. Aging Clin Exp Res. 2015;27(4):413-8. bone marrow mesenchymal stem cells isolated from cyclic and acyclic rats through different pathways (29). Both osteoblasts and osteoclasts express OTRs. Oxytocin stimulates osteoblast differentiation and also modulates osteoclast formation and function (30-31). It may promote osteoclastic differentiation; however, it prevents the resorptive action of mature osteoclasts. Oxytocin might also inhibit bone resorption and states in which OT decreases the RANKL/OPG ratio. It also causes the release of intracellular calcium leading to a calcium increase in osteoclast cells, which in turn increases nitric oxide synthesis as a mechanism to inhibit bone resorption (32).

In conclusion, our results strongly supports the hypothesis that ovariectomy -induced bone loss is related to low levels of circulating OT. Furthermore, Maintaining OT levels early after decline of ovarian functions holds promise as a potential protective measure against bone loss.

Further oxytocin-related studies on bone are likely to identify the most efficient OT dosage that can protect against postmenopausal OP, and potential side effects. In addition, OT half-life is relatively short, characterization of stable OT analogs with longer half-lives, such as carbetocin (33) or conjugated OT (34) may aid in the development of beneficial treatments.

\section{Acknowledgment}

The authors declare that there is no conflict of interest. This work was funded by Sohag Faculty of Medicine.

We appreciate the help of the Animal Facility workers of Sohag Faculty of Medicine in taking care of the rats.

3. Kuo TR, Chen $\mathrm{CH}$. Bone biomarker for the clinical assessment of osteoporosis: recent developments and future perspectives. Biomark Res. 2017;5:18.

4. Mendis S, Puska P, Norrving B. Global atlas on cardiovascular disease prevention and control. World Heart Federation; World Stroke Organization. 2011

5. Parra-Torres AY, Valdes-Flores M, Orozco L, Velazquez-Cruz R. Molecular Aspects of Bone 
Remodeling. Topics in Osteoporosis. 2013.

6. Schorr M, Marengi DA, Pulumo RL, Yu E, Eddy KT, Klibanski A, et al. Oxytocin and Its Relationship to Body Composition, Bone Mineral Density, and Hip Geometry Across the Weight Spectrum. J Clin Endocrinol Metab. 2017;102(8):2814-2824.

7. Colaianni G, Tamma R, Di Benedetto A, Yuen T, Sun L, Zaidi M, et al. The oxytocin-bone axis. J Neuroendocrinol. 2014;26(2): 53-7.

8. Colaianni G, Sun L, Di Benedetto A, Tamma $\mathrm{R}$, Zhu LL, Cao J, et al. Bone marrow oxytocin mediates the anabolic action of estrogen on the skeleton. J Biol Chem. 2012;287(34): 29159-67.

9. Weigt C, Hertrampf T, Zoth N, Fritzemeier KH, Diel P. Impact of estradiol. ER subtype specific agonists and genistein on energy homeostasis in a rat model of nutrition induced obesity. Mol Cell Endocrinol. 2012;351(2): 227-38.

10.Vickers MR, MacLennan AH, Lawton B, Ford D, Martin J, Meredith SK, et al. Main morbidities recorded in the women's international study of long duration oestrogen after menopause (WISDOM): a randomised controlled trial of hormone replacement therapy in postmenopausal women. BMJ., 2007;335(7613):239.

11.Colaianni G, Sun L, Zaidi M, Zallone A. Oxytocin and bone. Am J Physiol Regul Integr Comp Physiol. 2014;307(8):R970-7.

12.Alessandri N, Piccioni M, Isabelli V, Alessandri G, Di Matteo A, Padovani D, Rondoni G, Camardella B, Parlapiano C. Morphological and functional changes of cardiovascular system in postmenopausal women. Eur Rev Med Pharmacol Sci. 2007;11(2):107-117.

13.Beranger G.E, Pisani DF, Castel J, Djedaini M, Battaglia S, Amiaud J, Boukhechba F, Ailhaud G, Michiels JF, Heymann D, Luquet S. Oxytocin reverses ovariectomy-induced osteopenia and body fat gain. Endocrinology. 2014;155(4):1340-1352.

14. Boyce BF, Xing L. Biology of RANK, RANKL, and osteoprotegerin. Arthritis Res Ther. 2007. 9(Suppl1).

15.Xu C. RANKL-RANK signaling regulates osteoblast differentiation and bone formation. Bone Res. 2018;6:35.

16.Fallahnezhad S, Piryaei A, Darbandi H, Amini A, Ghoreishi SK, Jalalifirouzkouhi R, et al. Effect of low-level laser therapy and oxytocin on osteoporotic bone marrow-derived mesenchymal stem cells. J Cell Biochem. 2018;119(1): 983-997.

17. Khajuria DK, Razdan R, Mahapatra DR. Description of a new method of ovariectomy in female rats. Rev Bras Reumatol. 2012;52(3):462-70. 18.Beranger GE, Djedaini M, Battaglia S, Roux CH, Scheideler M, Heymann D, Amri EZ, Pisani DF. Oxytocin reverses osteoporosis in a sexdependent manner. Front Endocrinol (Lausanne). 2015;6:81.

19.Levin V. A., Jiang X., Kagan. Estrogen therapy for osteoporosis in the modern era. Osteoporos Int. 2018;29:1049-1055.

20.Li CW, Liang B, Shi XL, Wang H. Opg/Rankl mRNA dynamic expression in the bone tissue of ovariectomized rats with osteoporosis. Genet Mol Res. 14(3):9215-9224.

21.Kalaiselvi VS, Prabhu K, Ramesh M. Venkatesan V. Association of Serum Osteocalcin with Bone Mineral Density in Postmenopausal Women. J Clin Diagn Res. 2013;7(5):814-816.

22. Grigoryan AV, Dimitrova AA, Kostov KG, Russeva AL, Atanasova MA, Blagev AB, et al. Changes of serum concentrations of alkaline phosphatase and metalloproteinase-9 in an ovariectomized wistar rat model of osteoporosis. J Biomed Clin Res. 2017;10(1):32-36.

23. Rogers A, Saleh G, Hannon RA, Greenfeld D, Eastell R. Circulating estradiol and osteoprotegerin as determinants of bone turnover and bone density in postmenopausal women. J Clin Endocrinol Metab. 2002;87(10):4470-5.

24.Li X, Niu QT, Warmington KS, Asuncion FJ, Dwyer D, Grisanti M, et al. Progressive increases in bone mass and bone strength in an ovariectomized rat model of osteoporosis after 26 weeks of treatment with a sclerostin antibody. Endocrinology. 2014;155(12):4785-97.

25.Li L, Chen X, Lv S, Dong M, Zhang L, Tu J, et al. Influence of Exercise on Bone RemodelingRelated Hormones and Cytokines in Ovariectomized Rats: A Model of Postmenopausal Osteoporosis. PLoS One. 2014;9(11):e112845.

26.Feresin RG, Johnson S A, Elam ML, Kim JS, Khalil DA, Lucas EA, et al. Effects of Vitamin e on Bone Biomechanical and Histomorphometric Parameters in Ovariectomized Rats. J Osteoporosis. 2013:825985. 
27. Yamagishi T, Kawashima H, Ogose A, Ariizumi T, Oike N, Sasaki T, et al. Expression Profiling of Receptor-Activator of Nuclear Factor Kappa B Ligand in Soft Tissue Tumors. Tohoku J Exp Med. 2019;248(2):87-97.

28.Tulay Okman-Kilic. Estrogen Deficiency and Osteoporosis. Advances in Osteoporosis. 2015 29.Perilli E, Le V, Ma B, Salmon P, Reynolds K, Fazzalari NL Detecting early bone changes using in vivo micro-CT in ovariectomized, zoledronic acid-treated, and sham-operated rats. Osteoporos Int. 2010;21(8):1371-82.

30.Santos LF, Singulani MP, Stringhetta-Garcia CT, Oliveira SHP, Chaves-Neto AH, Dornelles RCM. Oxytocin effects on osteoblastic differentiation of Bone Marrow Mesenchymal Stem Cells from adult and aging female wistar rats. Exp Gerontol. 2018;113:58-63.

31.Tamma R, Colaianni G, Zhu LL, DiBenedetto A, Greco G, Montemurro G, et al. Oxytocin is an anabolic bone hormone. Proc Natl Acad Sci U S A. 2009;106(17):7149-54.

32.Kim SH, MacIntyre DA, Firmino Da Silva M, Andrew M Blanks 2, Yun S Lee 1, Steven Thornton et al. Oxytocin activates NF-kBmediated inflammatory pathways in human gestational tissues. Mol Cell Endocrinol. 2015;403:64-77.

33.Jin B, Du Y, Zhang F, Zhang K, Wang L, Cui L. Carbetocin for the prevention of postpartum hemorrhage: a systematic review and metaanalysis of randomized controlled trials. J Matern Fetal Neonatal Med 2016;29(3):400-7.

34.Cavallaro G, Maniscalco L, Campisi M, Schillaci D, Giammona G. Synthesis, characterization and in vitro cytotoxicity studies of a macromolecular conjugate of paclitaxel bearing oxytocin as targeting moiety. European Journal of Pharmaceutics and Biopharmaceutics. 2007;66(2):182-192. 